\title{
Handel und Innovation: Chance oder Gefahr
}

\section{Céline Diebold}

\section{Relevanz}

Handel und Innovation lösen einen starken Strukturwandel aus. Sie sind Chance und Gefahr zugleich. Innovative Unternehmen erschliessen in China und anderswo neue Märkte, expandieren und schaffen mehr Beschäftigung. Andere müssen der Importkonkurrenz weichen. Die Konsumenten profitieren von günstigen Preisen und einer grösseren Auswahl. Handel und Innovation ermöglichen grosse Wohlstandsgewinne, aber nur, wenn Arbeit und Kapital erfolgreich von schrumpfenden zu expandierenden Unternehmen wandern. Wie ist inklusives Wachstum möglich, an dem möglichst alle teilhaben können? Den Strukturwandel aufzuhalten würde den Fortschritt blockieren. Die Politik braucht ein Programm, das die besonders betroffenen Arbeitenden absichert und darin aktiv unterstützt, mit neuen Qualifikationen anderswo eine neue Beschäftigung mit besseren Perspektiven zu finden.

\section{Quelle}

Autor, David H., David Dorn und Gordon H. Hanson (2015), Untangling Trade and Technology: Evidence from Local Labor Markets, Economic Journal 125, 621-646.

Globalisierung und technologischer Fortschritt verändern die Arbeit und erfordern neue Qualifikationen. Technologische Neuerungen ermöglichen es

C. Diebold $(\bowtie)$

Universität St.Gallen, St.Gallen, Schweiz

E-Mail: celine.diebold@unisg.ch 
den Unternehmen, produktiver zu werden, etwa indem sie Routineaufgaben computerbasiert automatisieren. Dies hat grosse Folgen für den Arbeitsmarkt: Zum einen fallen die betroffenen Arbeitsplätze weg, zum anderen kann die Nachfrage nach Fachkräften wie z. B. IT-Spezialisten steigen. Auch der zunehmende internationale Handel löst einen Strukturwandel aus und verändert die Beschäftigungsverhältnisse nachhaltig. Im Wettbewerb mit Niedriglohnländern geraten Löhne und Arbeitsplätze in den Industriestaaten zunehmend unter Druck.

Wie technologischer Fortschritt und Welthandel die Beschäftigung beeinflussen, erforschen die Ökonomen schon seit langem, aber meist getrennt voneinander. David Autor, Gordon Hanson und David Dorn vergleichen die Auswirkungen der beiden Trends miteinander. Sind die Umwälzungen auf dem Arbeitsmarkt eher eine Folge des internationalen Handels oder des technologischen Fortschritts? Wie unterscheiden sich Technologie und Handel im Hinblick auf verschiedene Gruppen von Arbeitnehmern, Tätigkeiten und Branchen?

Die Forscher schätzen, wie sich Innovation und Handel auf die Beschäftigung in den USA zwischen 1980 und 2007 ausgewirkt haben. Dabei definieren sie 722 regionale Arbeitsmärkte, welche sich in der Bedeutung verschiedener Wirtschaftszweige unterscheiden. Je nach Branchenstruktur sind sie also mit unterschiedlicher Intensität dem internationalen Wettbewerb und technologischen Wandel ausgesetzt.

Die Folgen der Innovation für die Beschäftigung hängen sehr von der Routineintensität der Arbeitsplätze ab. Routineaufgaben sind besonders leicht zu automatisieren. Dies trifft beispielsweise auf Produktionsvorgänge, Büroarbeiten oder kontrollierende Tätigkeiten zu, jedoch weniger auf abstrakte (z. B. Mitarbeiterführung) oder handwerkliche Tätigkeiten. Dagegen hängt der internationale Wettbewerbsdruck auf die Beschäftigung in amerikanischen Unternehmen sehr mit dem Aufstieg Chinas zusammen. Seine Bedeutung im weltweiten Handel hat massiv zugenommen. China zählt heute zu den wichtigsten Handelspartnern der USA. Die Forscher nutzen Daten über diese beiden Entwicklungen in jedem der über 700 regionalen Arbeitsmärkte und quantifizieren die Beschäftigungseffekte von Technologie und Handel, indem sie auf die Routineintensität eines typischen Arbeitsplatzes bzw. auf die Zunahme von US-Importe aus China pro Arbeitsplatz abstellen.

Wie beeinflussen Handel und technologischer Fortschritt die Beschäftigungsund Arbeitslosenzahlen? Werden heimische Beschäftigte verdrängt? Die Schätzungen ergeben, dass die Automatisierung von Routineaufgaben insgesamt zu keinem Rückgang der Beschäftigungsquote führte, aber der internationale Wettbewerbsdruck aufgrund steigender Importe aus China signifikante Beschäftigungsverluste auslöste. Dabei gibt es grosse regionale Unterschiede in der Importkonkurrenz. Die Importe waren im obersten Quartil der regionalen Arbeitsmärkte mit der höchsten Importintensität um rund 1'100 US\$ pro 
Beschäftigten höher als im untersten Quartil der Regionen mit dem geringsten Importanteil. Die Wissenschaftler schätzen, dass ein Anstieg der Importe aus China um 1'000 US\$ pro Arbeitnehmer über zehn Jahre mit einem Rückgang der Beschäftigungsquote um 0,7 Prozentpunkte sowie mit einem Anstieg der Arbeitslosen- und Nichterwerbsquote um 0,2 bzw. 0,5 Prozentpunkte einherging. Der Beschäftigungsrückgang führte in rund drei von vier Fällen dazu, dass die Betroffenen dauerhaft aus dem Arbeitsmarkt ausschieden. Nur wenige wechselten in die (temporäre) Arbeitslosigkeit.

Um 1'000 US\$ höhere Importe aus China pro Arbeitnehmer verringerten die Beschäftigungsquote in den USA um 0.7 Prozentpunkte in 10 Jahren, wogegen die Automatisierung weder zu Beschäftigungsgewinnen noch Verlusten führte.

Dabei sind verschiedene demographische Gruppen einem recht unterschiedlichen Risiko ausgesetzt. Die Schätzungen zeigen, dass der technologische Fortschritt die Beschäftigungsquote von Frauen und älteren Arbeitnehmern signifikant verringerte. Diese Gruppen waren überproportional in Berufen mit vielen leicht automatisierbaren Routineaufgaben tätig. Die stärkere Konkurrenz aus China betraf hingegen alle Bildungs-, Geschlechts- und Altersgruppen. Gering qualifizierte Arbeitnehmer mussten die stärksten Beschäftigungsverluste hinnehmen. Ein Importanstieg von 1'000 US\$ pro Arbeitnehmer führte zu einem Rückgang der Beschäftigungsquote um 1,2 Prozentpunkte bei niedrig qualifizierten Arbeitnehmern, aber nur um 0,5 Prozentpunkte bei höher Qualifizierten. In beiden Fällen schieden die betroffenen Arbeitnehmer oft ganz aus dem Arbeitsmarkt aus. Die Forscher weisen jedoch darauf hin, dass dies an den verwendeten Daten liegen könnte, welche alle sieben bzw. zehn Jahre erhoben wurden. So werden nur mittelfristige Auswirkungen auf die Beschäftigungszahlen erfasst. Es ist daher vorstellbar, dass Arbeitnehmer zunächst arbeitslos wurden, bevor sie schliesslich den Arbeitsmarkt verliessen.

Die zunehmende Importkonkurrenz aus China traf niedrig qualifizierte Arbeitnehmer besonders stark. Die Beschäftigungsquote sank um 1.2 Prozentpunkte je 1'000 US\$ Importe pro Arbeitnehmer, verglichen mit nur 0.5 Prozentpunkten bei höher Qualifizierten.

Welche Berufsgruppen und Aufgabenfelder sind am stärksten von Technologieentwicklung und internationalem Wettbewerb betroffen? Die Forscher unterscheiden grob zwischen drei Kategorien: Tätigkeiten in Management und Technik verlangen spezialisierte Fähigkeiten in Organisation und abstrakter 
Problemlösung. Sie werden meist von gutbezahlten Arbeitnehmern mit hohem Bildungsstand ausgeführt. Tätigkeiten in Produktion, Administration und Vertrieb umfassen typischerweise zahlreiche Routineaufgaben, welche relativ leicht durch Computer ersetzbar bzw. automatisierbar sind. Eine dritte Kategorie bilden Berufe in Handwerk, Landwirtschaft und Dienstleistungen, welche oft körperliche Arbeit mit sich bringen und keine höhere Ausbildung voraussetzen. Sie haben sich meist als nur schwer automatisierbar erwiesen.

Zunehmende Automatisierung betrifft vor allem die zweite Kategorie mit hoher Routineintensität und führte dort zu signifikant niedrigeren Beschäftigungsquoten. Dies geschah unabhängig von Alter, Geschlecht sowie Ausbildung der Arbeitnehmer. Im Gegensatz dazu veränderte sich die Beschäftigungsquote von hoch bzw. niedrig qualifizierten Tätigkeiten (erste und dritte Kategorie) nicht signifikant. Automatisierung und Computerisierung tragen also zu einer Polarisierung von Berufen bei. So gehen zwar Arbeitsplätze etwa in Verwaltung und Büro verloren. Es entstehen aber neue Arbeitsplätze sowohl in leitenden Tätigkeiten als auch in handwerklichen Berufen, welche die entgegensetzten Enden des Einkommensspektrums besetzen. Diese Entwicklung erklärt zumindest teilweise die immer größer werdende Einkommensungleichheit in den USA. Der zunehmende Wettbewerb mit China führte dagegen zu Beschäftigungsverlusten in allen Berufskategorien, mit überdurchschnittlich starken Auswirkungen auf Arbeitnehmer mit nur geringen Qualifikationen oder in routineintensiven Tätigkeiten.

Die Beschäftigungsquote in Produktion, Administration und Vertrieb sank um 1.8 Prozentpunkte stärker, wenn diese Tätigkeiten besonders routineintensiv und leicht automatisierbar waren.

Schliesslich vergleichen die Forscher die Entwicklung in verschiedenen Branchen. In der Industrie führte zunehmende Konkurrenz aus China zu deutlichen Arbeitsplatzverlusten: Die Beschäftigungsquote sank über zehn Jahre um 0,5 Prozentpunkte, wenn chinesische Importe um 1'000 US\$ pro Beschäftigten zunahmen. Von diesem Rückgang waren nicht nur Mitarbeiter in der Produktion selbst, sondern auch leitende und technische Angestellte sowie Büromitarbeiter betroffen. Dagegen verursachte der technologische Fortschritt im gesamten Beobachtungszeitraum von 1980 bis 2007 insgesamt kaum signifikante Arbeitsplatzverluste in der Industrie. Aber die Verwendung von Computern, vor allem in den 1980er und 1990er Jahren, veränderte Aufgabenfelder und Berufsbilder sehr stark, führte zu Beschäftigungsverlusten bei routineintensiven Tätigkeiten, und förderte eine Polarisierung der Arbeitswelt. Dieser Effekt nahm in den 2000er Jahren deutlich ab. In anderen Branchen hingegen nahm die Automatisierung von Routinetätigkeiten vor allem nach dem Jahr 2000 noch weiter zu. 


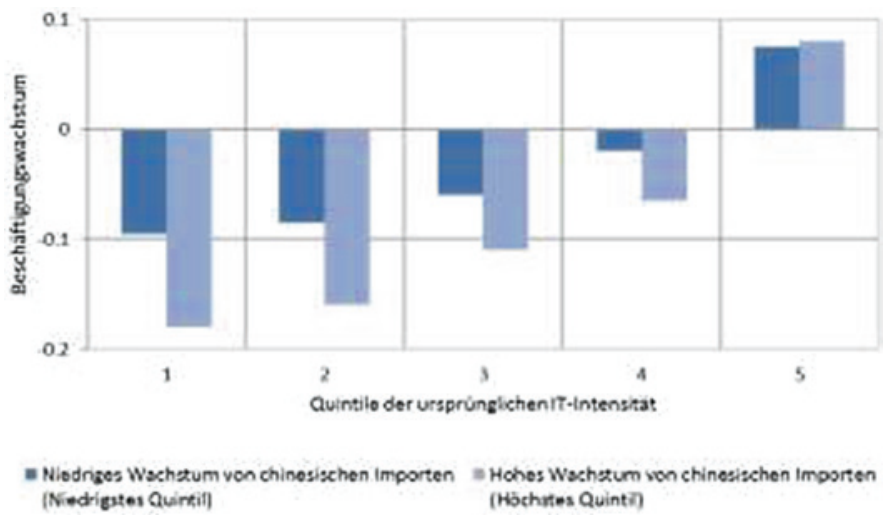

Abb. 1 Beschäftigungseffekt unterschiedlich starker chinesischer Importkonkurrenz in 12 EU-Staaten. Bemerkung: Beschäftigungswachstum je nach Innovationsgrad der Firmen in Branchen mit schwach und stark steigenden Importen aus China. Die IT-Intensität ist von Quintil 1 zu 5 ansteigend. (Quelle: Nicholas Bloom, Mirko Draca und John Van Reenen, Who's Afraid of the Big Bad Dragon? How Chinese Trade Boosts European Innovation, VOXeu, 3. Februar 2011)

In der Industrie stellt seit den 1990er Jahren die Importkonkurrenz aus China eine immer grösser werdende Gefahr für die Beschäftigung dar. Der Trend zur Automatisierung führt weniger in der Industrie, aber zunehmend im Dienstleistungssektor zu Arbeitsplatzverlusten.

Die Auswirkungen des technologischen Fortschritts auf den amerikanischen Arbeitsmarkt scheinen ihren Fokus zu verschieben: Während in der Vergangenheit Produktionsabläufe automatisiert und hierdurch Arbeitsplätze abgebaut wurden, war zwei Jahrzehnte später der Dienstleistungssektor betroffen, z. B. durch Neuerungen in der Daten- und Informationsverarbeitung.

In einer ähnlichen Studie untersuchen Nicholas Bloom und seine Co-Autoren ${ }^{1}$ die Folgen der chinesischen Importkonkurrenz für zwölf europäische Staaten im Zeitraum von 1996 bis 2007. Abb. 1 veranschaulicht den Beschäftigungszuwachs bzw. -rückgang europäischer Firmen in Sektoren, welche in einem schwachen

${ }^{1}$ Nicholas Bloom, Mirko Draca und John van Reenen (2016), Trade Induced Technical Change? The Impact of Chinese Imports on Innovation, IT and Productivity, Review of Economic Studies 83, 87-117. 
bzw. starken Ausmaß von chinesischer Importkonkurrenz betroffen waren, je nach Technologieintensität der Unternehmen. Firmen mit geringer IT-Intensität schrumpften überall, aber besonders stark in Branchen und Regionen mit hoher chinesischer Importkonkurrenz. Relativ IT-intensive Unternehmen konnten jedoch offenbar unabhängig vom Ausmaß der chinesischen Importkonkurrenz einen Zuwachs der Beschäftigungszahlen verzeichnen. Dies deutet darauf hin, dass zwar wenige produktive Firmen im Zuge der chinesischen Importe verdrängt wurden. Innovative und technologieintensive Unternehmen konnten jedoch erfolgreich reagieren und ihre Marktanteile, unbeeindruckt der chinesischen Konkurrenz, sogar ausbauen.

Open Access Dieses Kapitel wird unter der Creative Commons Namensnennung 4.0 International Lizenz (http://creativecommons.org/licenses/by/4.0/deed.de) veröffentlicht, welche die Nutzung, Vervielfältigung, Bearbeitung, Verbreitung und Wiedergabe in jeglichem Medium und Format erlaubt, sofern Sie den/die ursprünglichen Autor(en) und die Quelle ordnungsgemäß nennen, einen Link zur Creative Commons Lizenz beifügen und angeben, ob Änderungen vorgenommen wurden.

Die in diesem Kapitel enthaltenen Bilder und sonstiges Drittmaterial unterliegen ebenfalls der genannten Creative Commons Lizenz, sofern sich aus der Abbildungslegende nichts anderes ergibt. Sofern das betreffende Material nicht unter der genannten Creative Commons Lizenz steht und die betreffende Handlung nicht nach gesetzlichen Vorschriften erlaubt ist, ist für die oben aufgeführten Weiterverwendungen des Materials die Einwilligung des jeweiligen Rechteinhabers einzuholen.

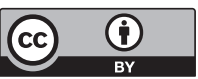

